\title{
Analysis of Individual Faith through Holy Bible Using Fuzzy Clustering
}

\author{
A. Victor Devadoss, A. Rajkumar, and C. Jayalatha
}

\begin{abstract}
As the world scenario has been witnessing different types of natural catastrophes, loss of human life, destruction of sustenance, people have grown cold and have lost hope. In an attempt to revive this lost faith, this paper studies the survey done on Individual Faith as identified from various incidences in the Holy Bible. Fuzzy Clustering which enables classification of data elements into different clusters based on varying degrees of a selected character was the selected methodology. Applying this, the events or data elements were grouped into various clusters based on varying degrees of Faith, on a scale from one to ten, i.e., High, Moderate and Low cluster groups supported by expert opinion on each category. Since clustering algorithms are useful in situations where little prior knowledge exists, this concept was applied to make a quantitative comparison of various degrees of faith.
\end{abstract}

Index Terms-Clustering algorithms, lost faith, degrees of faith, Unsupervised, Bible.

\section{INTRODUCTION}

In these end times, catastrophes, annihilation and destruction are on the increase and have shattered man's beliefs. Mankind is in need of immense Grace given from above. Dispensation of this grace and in abundance is dependent on the faith vested in an individual. Faith in totality has two elements ie., being convinced of the truth, being certain of reality, having evidence of unseen things, and believing, hoping in, embracing, seizing the truth(Heb 11:1 (KJV) Now faith is the substance of things hoped for, the evidence of things not seen).This study has identified various wonderful incidents in the Holy scriptures which stand testament, that Faith, is required if not to a greater degree, to have hope and to make us certain of the realities we do not see. The methodology selected for this study is Fuzzy Clustering. The clustering methods divide a set of $N$ observations (input vectors) $\boldsymbol{x}_{1}, \boldsymbol{x}_{2}, \ldots, \boldsymbol{x}_{N}$ into $c$ groups denoted by $1 ; 2 ;: \therefore ; c$ in such a way that the members of the same group are more similar to one another than to the members of other groups. The number of clusters may be pre-de_ned or it may be set by the method.Generally, clustering methods can be divided into (Duda and Hart, 1973; Fukunaga, 1990; Tou and Gonzalez, 1974) the following kinds: hierarchical, graph theoretic, decomposing a density function, minimizing a criterion function. In this paper, clustering by minimization of a criterion function will be considered. Usually, the clustering methods assume that each data vector belongs to one and only one class. This method

Manuscript received November 15, 2012; revised January 29, 2013.

A. Victor Devadoss is with the Loyola College Nugabakkam Chennai-600034, India (grace238@gmail.com).

A. Rajkumar and J. Jayalatha are with the Hindustan University, Padur, chennai 603103, India (arajkumar@hindustanuniv.ac.in, jayalatha@hindustanuniv.ac.in). can be natural for clustering of compact and well-separated groups of data. Fuzzy clustering is more appropriate for perceptions based data since perceptions are always a matter of varying degree. The organization of this article is as follows: Choosing the attributes in the Methodology section, Basic notion of clustering and fuzzy clustering in following section, Results and Discussion in the third section and finally the conclusion and scope for future study is presented in the fourth and final section.

\section{MethodolOGY}

In this methodology, the aim is to find the faith of an individual for which the following twenty attributes of individual faith has been selected by interviewing the religious people in Chennai belonging to different ages [1].

1. Arresting of Jesus : John:18-25-27, Peter's faith

2. Changing water into wine, John 2:1-11-Mary's faith

3. Healing of the woman with internal bleeding, Mathew 9:20-22, a woman's faith

4. Healing of the centurions servant, Mathew:8:5-13, the centurions faith

5. The faith of Thomas, John 20:24-25,

6. Raising of Lazarus, Martha's faith

7. The Birth of Jesus Christ, Mary's faith

8. Walking on water, Peter's faith

9. Healing the two blind men - the blind man's faith

10. Catching a large number of fish, Simon Peter's faith

11. The Birth of John, the Baptist, elizebeth faith

12. Calming the stormy sea- the disciples' faith

13. Cathing a fish with a coin in its mouth, Peter's faith

14. The faith of juda when he hanged himself.

15. Healing Bartimaeous of blindness

16. Healing a deaf man with speech impediment

17. Repentance of one of the thieves crucified along with Jesus Christ

18. Non- repentance of one of the thieves at the cross

19. Mary Magdalene's faith

20. The faith of Juda

Three attributes which best define the characteristic of each segment were selected to be rated by respondents. Fuzzy c-means clustering is done using the algorithm (4.1).

\section{PRELIMINARIES}

\section{A. Hard Clustering [2]}

In Hard Clustering we make a hard partition of the data set 
$Z$. In other words, we divide them into $c \geq 2$ clusters. With a partition, we mean that

$$
\bigcup_{i=1}^{c} A_{i}=Z
$$

and $A_{i} \cap A_{j}==\phi, \forall i \neq j$

Also, none of the sets, $A_{i}$ may be empty. To indicate a portioning, we make use of membership functions $\mu_{k}(x)$. If $\mu_{k}(x)=1$, then object $x$ is in cluster $k$. Based on the membership functions, we can assemble the Partition Matrix $U$, of which $\mu_{k}(x)$ are the elements. Finally there is a rule that $\forall x$,

$$
\sum_{i=1}^{c} \mu_{k} \quad(x)=1 \forall x
$$

In other words, every object is only part of one cluster.

\section{B. Fuzzy Clustering}

Hard clustering has a downside. When an object roughly falls between two clusters $A_{i}$ and $A_{j}$, it has to be put into one of these clusters. Also, outliers have to be put in some cluster. This is undesirable. But it can be fixed by fuzzy clustering.

In Fuzzy clustering, we make a Fuzzy partition of the data. Now, the membership function $\mu_{k}(x)$ can be any value between 0 and 1 . This means that an object $z_{k}$ can be for 0.2 parts in $A_{i}$ and for 0.8 parts in $A_{j}$. However, requirement (2) still applies. So, the sum of the membership functions still has to be 1 . The set of all fuzzy partitions that can be formed in this way is denoted by $M_{f c}$. Fuzzy partitioning again has a downside. When we have an outlier in the data (being an object that doesn't really belong to any cluster), we still have to assign it to clusters. That is, the sum of its membership functions still must equal one.

\section{Fuzzy C-Means Clustering [3]}

In fuzzy clustering, each point has a degree of belonging to clusters, as in fuzzy logic, rather than belonging completely to just one cluster. Thus, points on the edge of a cluster, may be in a cluster to a lesser degree than points in the center of cluster for each point $x$ there is no coefficient giving the degree of belonging in the $k^{\text {th }}$ cluster $\mu_{\mathrm{k}}(x)=1$. Usually, the sum of those coefficients is defined to be 1 .

$$
\sum_{n=1}^{m m} \mu_{k=1} \forall x
$$

With fuzzy c-means, the centroid of a cluster is the mean of all points, weighted by their degree of belonging to the cluster.

$$
\text { Center }_{k}=\frac{\sum_{x} \mu_{k}(x)^{m} x}{\sum_{x} \mu_{k}(x)^{m}}
$$

The degree of belonging is related to the inverse of the distance to the cluster

$$
\mu_{K}(x)=\frac{1}{d\left(\text { center }_{k}, x\right)^{\prime}}
$$

Then, the coefficients are normalized and fuzzy field with a real parameter $m>1$ so that their sum is 1 . So

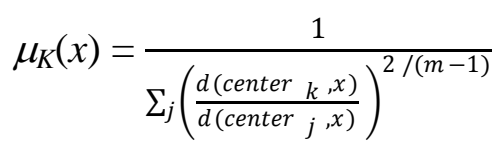

For $m$ equal to 2 , this is equivalent to normalizing the coefficient linearly to make their sum 1 . When $\mathrm{m}$ is close to 1 , then cluster center closest to the point is given much more weightage than the others, and the algorithm is similar to $k$-means [4], [5].

\section{RESULTS AND DISCUSSION}

A number of religious people in Chennai city were interviewed in an attempt to measure the faith of an individual with the Holy Bible as a reference. From these, twenty attributes were chosen and the respondents had related the attributes of problems engendered high level faith on a 10-point rating scale and the results of the average rating scale and the results of the average rating are shown in Fig.1 $2^{\text {nd }}$ and $9^{\text {th }}$ attributes is rated highest by the religious people and clergy with an average rating of 8.5 and 8.3 respectively on a 10-point scale. This means that, on comparison with other attributes, the $2^{\text {nd }}$ and $9^{\text {th }}$ attributes engendered high level faith and the $14^{\text {th }}$ attribute which was rated an average 2.8 was engendered as a low level faith.

Attributes of an individual faith

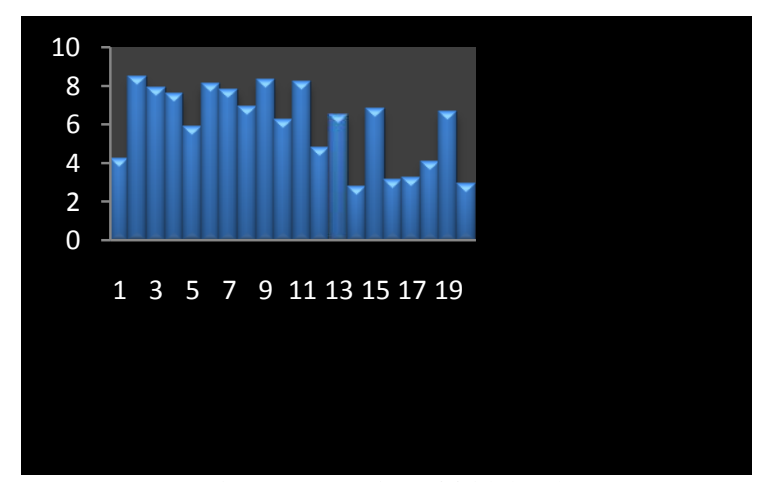

Fig. 1. Mean rating of faith level

The ratings and the Standard Deviation of the attributes of the individual faith analyzed by religious people engendered high level frustration have been subjected to fuzzy c-means clustering using the algorithm: 1 and the following results shown in Table I have been obtained for a 3-cluster combination. The first cluster comprises of the attributes with average rating from 2.7 to 5.7 with a mid value 4.2 . The second cluster range is from 3.0 to 7.0 with a mid valued 5 and the third cluster has a range of 6.0 to 10 with a mid value 8.

TABLE I: 3-CLUSTER RANGE OF LEVEL OF FAITH

\begin{tabular}{||l|c|c|c||}
\hline & Cluster 1 & Cluster 2 & Cluster 3 \\
\hline Range & $2.7-5.7$ & $3.0-7.0$ & $6.0-10$ \\
Mid- Value & 4.2 & 5 & 8 \\
Classification & LOW & MODERATE & HIGH \\
\hline
\end{tabular}


The first cluster range indicates the faith of an individual which engendered LOW level faith. Second and Third clusters range show the MODERATE and HIGH level of faith respectively. There are Over-lapping ranges as is characteristic of a fuzzy based cluster [6], [7].

\section{Algorithm to Find a Membership Values for the Attributes [8]:}

\section{Step 1: Start}

Step 2: Fix, the values of 20 attributes on a 10-point rating scale in a set D (say)

Step 3: Fix the cluster, which is defined as Cluster $1=$ LOW, whose range beginning with 2.5 (bv1) End with 5.5(ev1). Cluster 2 = MODERATE, whose range beginning with 3.5 (bv2) end with 8.5(ev2). Cluster $3=\mathrm{HIGH}$, whose range beginning with 7.5 (bv3) end with 10 (ev3).

Step 4: Choose en element $x$ in D

Step 5: If $x<\operatorname{ev1,~Go~to~Step~6,~else~Go~to~Step8~}$

Step 6: If $x>$ bv2, then $x$ lies in cluster 1 and cluster 2 whose membership value is defined as $\mu_{k}(x)=\operatorname{ev1-x:} x$ - bv2, Go to Step 12, else Go to Step7.

Step 7: Then $x$ lies in cluster 1 only, the membership value is $\mu_{k}(x)=1$ Go to Step 12

Step 8: If $\boldsymbol{x}<\operatorname{ev2}$ Go to Step 9, else Go to Step 11

Step 9: If $x>$ bv3, then $x$ lies in cluster 2 and cluster 3, whose membership value is defined as $\mu_{k}(x)=$ ev2- $x: x$-bv3, Go to step 12, else Go to Step10

Step 10: Then $x$ lies in cluster 2 only, the membership value is $\mu_{k}(x)=1$ else Go to Step11

Step 11: Then $x$ lies in cluster 3 only, the membership value is $\mu_{k}(x)=1$

Step 12: Go to Step 4, until all the values in D have been checked

\section{Step 13: Stop}

Here 'bv' denotes the beginning value and 'ev' denotes the ending the value.

Degree of membership of the attributes that gives more faith of an individual is found using the above algorithm is shown in Table II. Attributes 14, 20 with a mean rating 2.8, 2.9 is entirely (100 percentage) with a membership value of lin cluster 1 .

(i.e.) faith level is LOW.

Attribute 5 with a mean rating 5.9 is entirely (100\%) with a membership value of 1 in cluster 2. (i.e.) faith level is MODERATE.

Attributes 1, 12,16,17\&18 with a mean ratings $4.2,4.8,3.1,3.2 \& 4.1$ belongs to $56 \%$ in cluster 1 and $44 \%$ in cluster $2,33 \%$ in cluster 1 and $67 \%$ in cluster $2,96 \%$ in cluster 1 and $4 \%$ in cluster $2,93 \%$ in cluster 1 and $7 \%$ in cluster 2, $59 \%$ in cluster 1 and $41 \%$ in cluster 2 . (i.e.) faith level is between MODERATE and HIGH.

Attributes 2, 3, 4 ,6, 7,8,9,10,11,13,15\&19 with a mean ratings $8.5,7.9,7.6,8.1,7.8,6.9,8.3,6.2,8.2,6.5,6.8 \& 6.7$ belongs to $4 \%$ in cluster 2 and $96 \%$ in cluster $3,27 \%$ in cluster 2 and $73 \%$ in cluster $3,38 \%$ in cluster 2 and $62 \%$ in cluster $3,19 \%$ in cluster 2 and $81 \%$ in cluster $3,31 \%$ in cluster 2 and $69 \%$ in cluster $3,65 \%$ in cluster 2 and $35 \%$ in cluster $3,12 \%$ in cluster 2 and $88 \%$ in cluster $3,92 \%$ in cluster 2 and $8 \%$ in cluster $3,15 \%$ in cluster 2 and $85 \%$ in cluster 3, $81 \%$ in cluster 2 and $19 \%$ in cluster $3,69 \%$ in cluster 2 and $31 \%$ in cluster $3 \& 73 \%$ in cluster 2 and $27 \%$ in cluster 3. (i.e.) faith level is between MODERATE and HIGH.

TABLE II: DEGREE OF MEMBERSHIP OF THE ATTRIBUTES

\begin{tabular}{||l|c|c|c|c||}
\hline S. & MEAN & LOW & MODERATE & HIGH \\
& & & & \\
\hline 1 & 4.2 & 0.56 & 0.44 & 0 \\
2 & 8.5 & 0 & 0.04 & 0.96 \\
3 & 7.9 & 0 & 0.27 & 0.73 \\
4 & 7.6 & 0 & 0.38 & 0.62 \\
5 & 5.9 & 0 & 1 & 0 \\
6 & 8.1 & 0 & 0.19 & 0.81 \\
7 & 7.8 & 0 & 0.31 & 0.69 \\
8 & 6.9 & 0 & 0.65 & 0.35 \\
9 & 8.3 & 0 & 0.12 & 0.88 \\
10 & 6.2 & 0 & 0.92 & 0.08 \\
11 & 8.2 & 0 & 0.15 & 0.85 \\
12 & 4.8 & 0.33 & 0.67 & 0 \\
13 & 6.5 & 0 & 0.81 & 0.19 \\
14 & 2.8 & 1 & 0 & 0 \\
15 & 6.8 & 0 & 0.69 & 0.31 \\
16 & 3.1 & 0.96 & 0.04 & 0 \\
17 & 3.2 & 0.93 & 0.07 & 0 \\
18 & 4.1 & 0.59 & 0.41 & 0.27 \\
19 & 6.7 & 0 & 0.73 & 0 \\
20 & 2.9 & 1 & & 0 \\
\hline
\end{tabular}

\section{CONCLUSION}

Application of Fuzzy clustering enabled to sort out the degree of faith in individuals in various situations. The twenty attributes selected were classified based on people's opinion into low, moderate and high based on this methodology. On analyzing the degree of membership of these attributes, it was found that twelve of the twenty attributes were highly rated. What is significant about this observation is the higher the degree of faith greater is the extent of miracle. But what needs to be noted is that eight of the attributes fall in the low level which indicates that even an individual with a mustard level of faith could definitely expect a miracle. This reinforces an individual's faith and brings hope in a time when the world is thrown into chaos. The attributes selected showcases different degrees of Faith in different situations. These attributes may be taken as booster shots for an individual's faith and reinforcing their faith in the Almighty. Fuzzy Clustering has successfully been used to analyze and quantify an abstract emotion "Faith" in an individual.

\section{SUGGESTIONS}

Faith is your spiritual hand simply accepting what is offered Faith is not a possession. It is decision, action, believing. Faith is greatest when you are least aware of it, when you act like a trusting child.

Faith is letting god pick you up and carry you like a trusting child. Faith is leaving things to god Rom 10:17 says faith comes by hearing, and hearing by the word of god: Luke 17:5-6 If you faith as a mustard seed, you can say to this mulberry tree, be pulled up by the roots and be planted in the sea, and it would obey you: Let increase your faith and get miracles from God. 


\section{ACKNOWLEDGMENT}

The authors wish to thank Mr. Ashok Verghese, Director Hindustan University - Chennai, for his encouragement and support

\section{REFERENCES}

[1] The Holy Bible, The bible society of India.

[2] A. J. Zolotor, A. C. Denham, and A. Weil, Intimate partner violence, Prim Care 2009.

[3] A. Kaufmann, Introduction to the theory of fuzzy subsets, Academic Press, inc. (london) ltd, 1975.

[4] B. Kosko, "Hidden pattern in combined and adaptive knowledge networks," Proc. of the First, in Proc. IEEE International Conference on Neural Networks, vol. 86, pp. 377-398, 1988.

[5] B. Kosko, Neural networks and fuzzy systems: a dynamical system approach to machine intelligence, Prentice Hall of India, 1997.

[6] H. J. Zimmermann, Fuzzy set theory and its application, fourth Edition Springer, 2011.

[7] J. K. George and B. Yuan, Fuzzy sets and fuzzy logic: theory and applications, Prentice Hall of India.

[8] A. V. Devadoss and A. Felix, "Fuzzy Clustering of the Domestic Violence for the Degree of Suicide thought based on Married Women Perception by 2012," IJERA, pp. 1770-1773

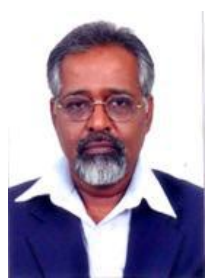

A. Victor Devadoss is the head and associate professor of PG \& Research Department of Mathematics, Loyola College, Chennai-34, Tamil Nadu, India. Director of Fr. Racine Research Centre, Loyola College, Chennai. He was deputed to BHUTAN under the Coloumbo plan 2007-2009. He has published more than 30 National \& International Journals. His Specialization area is Fuzzy Logic and its Application

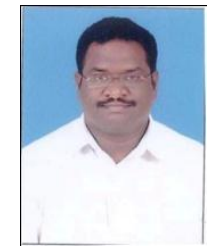

A. Rajkumar is a member of Research Promotion Committee and assistant professor of Dept Of Mathematics, Hindustan Institute of Science and Technology, Hindustan University, Padur, Chennai603.He has Ten years of teaching experience and two years of research experience. Currently research scholars (Ph.D) under his supervision and guidance of victor devadoss. His area of specilisation is Fuzzy Logic and models. He has published 12 articles in National and International Journals.

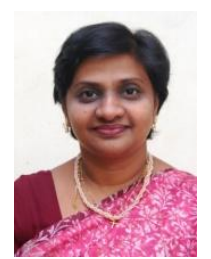

C. Jayalatha is currently serving as a professsor of Chemistry and head in charge of the Clean Energy and Nano Convergence Centre (CENCON) of Hindustan University (HITS). She is a M. Sc., M.Phil., PhD. holder in Polymer Chemistry from Anna University and has 21 years of teaching experience (19 in HITS). She has served as Former Head of the Departments of Polymer Technology and Science \& Humanities. During her tenure, she established the Organic chemistry Laboratory in the department and conducted an In-house workshop for second year polymer technology students titled "Preparation of Nano solutions" involving hands on training. Her areas of research interest are in Liquid Crystalline Polymers; She has guided Undergraduate projects in Polymer Technology and has 10 publications in International Journals and Conferences. 